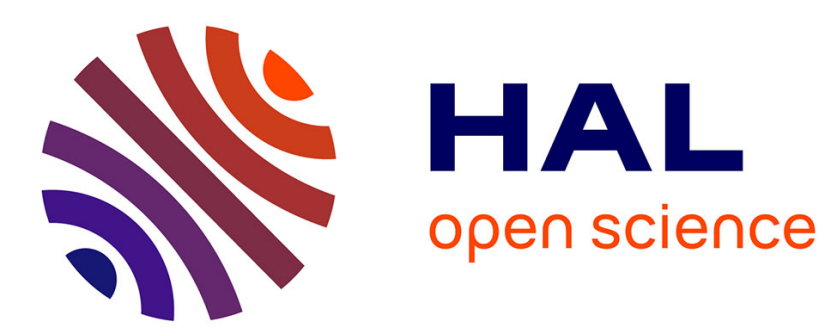

\title{
Structures Al-Al2O3-InP : Analyse des processus de dérive et évaluation des hauteurs de barrières par photoémission interne
}

\author{
S. Krawczyk, B. Sautreuil, P. Viktorovitch
}

\section{- To cite this version:}

S. Krawczyk, B. Sautreuil, P. Viktorovitch. Structures Al-Al2O3-InP : Analyse des processus de dérive et évaluation des hauteurs de barrières par photoémission interne. Revue de Physique Appliquée, 1983, 18 (12), pp.763-767. 10.1051/rphysap:019830018012076300 . jpa-00245144

HAL Id: jpa-00245144

https://hal.science/jpa-00245144

Submitted on 1 Jan 1983

HAL is a multi-disciplinary open access archive for the deposit and dissemination of scientific research documents, whether they are published or not. The documents may come from teaching and research institutions in France or abroad, or from public or private research centers.
L'archive ouverte pluridisciplinaire HAL, est destinée au dépôt et à la diffusion de documents scientifiques de niveau recherche, publiés ou non, émanant des établissements d'enseignement et de recherche français ou étrangers, des laboratoires publics ou privés. 


\title{
Structures Al- $\mathrm{Al}_{2} \mathbf{O}_{3}$-InP : Analyse des processus de dérive et évaluation des hauteurs de barrières par photoémission interne
}

\author{
S. Krawczyk, B. Sautreuil et P. Viktorovitch \\ Laboratoire d'Electronique, Automatique et Mesures Electriques de l'Ecole Centrale de Lyon $\left({ }^{*}\right)$, \\ 36, av. de Collongue, B.P. 163, 69131 Ecully Cedex, France
}

(Reçu le 27 juillet 1983, accepté le 5 septembre 1983)

\begin{abstract}
Résumé. - L'instabilité des caractéristiques électriques des structures MIS sur InP est actuellement un problème majeur pour le développement de ce type de dispositif et constitue la matière d'une polémique très ouverte dans la littérature. Dans ce travail, nous proposons une nouvelle approche de ces phénomènes fondée sur l'étude de la photoémission interne de structures $\mathrm{Al}-\mathrm{Al}_{2} \mathrm{O}_{3}$-InP. Cette technique permet, entre autres, la mesure directe de la hauteur de barrière semiconducteur-isolant et le contrôle du remplissage des pièges d'interface. $\mathrm{Al}_{2} \mathrm{O}_{3}$ est déposé par évaporation au canon à électrons. Avant le dépôt, les substrats d'InP subissent un polissage mécano-chimique $\mathrm{Br}-\mathrm{CH}_{3} \mathrm{OH}$, suivi de divers traitements chimiques conduisant soit au décapage de l'oxyde natif (solution $\mathrm{HF}$ ), soit à la constitution d'un oxyde stable (solution $\mathrm{NH}_{4} \mathrm{OH}$ ). En vue de comparaison, les mesures de photoémission sont aussi effectuées sur des structures $\mathrm{Al}-\mathrm{Al}_{2} \mathrm{O}_{3}-\mathrm{Si}$ dont l'isolant a été déposé dans les mêmes conditions, après avoir soumis le substrat de silicium à un traitement chimique classique. La hauteur de barrière $\chi_{n}$, présentée aux électrons de conduction de l'InP par l' $\mathrm{Al}_{2} \mathrm{O}_{3}(2,8 \pm 0,1 \mathrm{eV})$ est sensiblement plus faible que dans le cas du silicium $(3,1 \pm 0,1 \mathrm{eV})$. Les résultats ne dépendent pas, au premier ordre, du traitement chimique des substrats. La comparaison de ces résultats avec les valeurs rapportées dans la littérature de l'affinité électronique de l'InP et du Si, indique une contribution dipolaire relative de $0,65 \pm 0,1 \mathrm{eV}$ à l'interface $\mathrm{Al}_{2} \mathrm{O}_{3}$-InP par rapport à l'interface $\mathrm{Al}_{2} \mathrm{O}_{3}$-Si. La différence observée $(0,3 \mathrm{eV} \pm 0,1 \mathrm{eV})$ entre les valeurs de $\chi_{\mathrm{n}}$ pour l'InP et le Si n'est pas suffisante pour expliquer les phénomènes de dérive observés dans les structures MIS-InP sur la base de processus d'injection, suivis de piègeage, de porteurs chauds à l'intérieur de l'alumine. La corrélation des résultats de mesure de photoémission et des caractéristiques de dérive des dispositifs montre que ces dernières sont fortement tributaires de la qualité de l'isolant déposé et que leur dispersion, pouvant résulter d'une mauvaise reproductibilité des conditions de fabrication de l'isolant est considérablement réduite, de même que leur amplitude limitée, en présence d'un oxyde natif stable à la surface du semiconducteur.
\end{abstract}

\begin{abstract}
Drift phenomena of electrical characteristics of MIS devices on InP are presently a major difficulty for the development of this type of device and the matter of dispute in the literature. In this work, a novel analysis

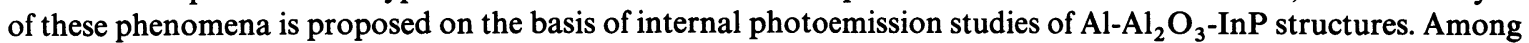
other applications, this technique gives access to the determination of the insulator-semiconductor barrier height and permits to control the filling of interface traps. $\mathrm{Al}_{2} \mathrm{O}_{3}$ is deposited by electron-gun evaporation. Prior to the deposition, InP substrates were prepared by first chemico-mechanically polishing them with a bromine-methanol solution followed by several chemical treatments resulting either in etching of the native oxide (HF solution) or in the formation of a stable oxide $\left(\mathrm{NH}_{4} \mathrm{OH}\right.$ solution). For comparison, photoemission measurements are also carried out on $\mathrm{Al}_{-} \mathrm{Al}_{2} \mathrm{O}_{3}$-Si structures whose insulator is deposited in the same conditions and silicon substrate submitted to a classical chemical treatment. The barrier height $\chi_{\mathrm{n}}$ which conduction electrons have to overcome to reach the $\mathrm{Al}_{2} \mathrm{O}_{3}$ conduction band is significantly lower for $\operatorname{InP}(2.8 \pm 0.1 \mathrm{eV})$ than for $\mathrm{Si}(3.1 \pm 0.1 \mathrm{eV})$. Results are practically independent of the chemical treatment of the semiconductor surface. The comparison of these results with the reported values of the electronic affinities of InP and $\mathrm{Si}$ reveals the contribution of an interface dipole of $0.65 \pm 0.1 \mathrm{eV}$ at the $\mathrm{Al}_{2} \mathrm{O}_{3}$-InP interface with regard to the $\mathrm{Al}_{2} \mathrm{O}_{3}-\mathrm{Si}$ interface. The difference $(0.3 \pm 0.1 \mathrm{eV})$ observed in the values of $\chi_{\mathrm{n}}$ for InP and Si is not sufficient to account for drift phenomena in InPMIS structures on the basis of the injection and trapping of hot electrons in $\mathrm{Al}_{2} \mathrm{O}_{3}$. The correlation between photoemission measurements and drifting behaviour of the devices shows that their stability depends strongly on the quality of the deposited insulator. It is further demonstrated that the formation of a stable native oxide results in the limitation of drift phenomena and in the reduction of the dispersion of electrical characteristics due to a poor reproductibility of the fabrication conditions of the gate insulator.
\end{abstract}

(*) E.R.A. du CNRS no 661 « Génie Electronique ». 


\section{Introduction.}

La fabrication de structures Métal-Isolant-Semiconducteur (MIS) sur InP permettant la formation d'un canal d'inversion d'électrons est apparue possible dès 1976 [1], alors que les multiples tentatives sur GaAs se sont avérées infructueuses jusqu'à ce jour. Ce résultat essentiel s'est cependant révélé insuffisant pour assurer le développement de transistors à effet de champ à grille isolée sur InP, développement rendu impossible pour un problème majeur non encore résolu : ces dispositifs sont en effet systématiquement le siège d'instabilités, se traduisant par des dérives lentes de leurs caractéristiques électriques. L'origine de ces phénomènes de dérive n'est pas encore établie et constitue la matière d'une polémique très ouverte dans la littérature. Cette polémique concerne en particulier la question de savoir si la présence ou non d'un oxyde natif d'InP situé entre le matériau semiconducteur et l'isolant de grille est souhaitable pour la stabilité du dispositif. Les avis sont très partagés à cet égard. A titre d'exemple, rappelons les travaux de $\mathrm{M}$. Okamura et al. $[2,3]$ publiés à un an d'intervalle et qui aboutissent à des conclusions opposées. Ces auteurs obtiennent successivement leurs meilleurs résultats en réalisant un décapage in situ par vapeur de $\mathrm{HCl}$ de l'oxyde natif [2], ou en favorisant la formation d'un oxyde thermique [3], dans les deux cas avant le dépôt de l'isolant de grille. Dans un article récent [4], D. Lile et ses collègues observent que les meilleurs résultats sont obtenus lorsque les substrats ne subissent aucune attaque chimique particulière. Dans un travail récent [5], nous montrons que la présence d'un oxyde natif de bonne qualité permet de neutraliser les états rapides de surface et s'avère donc indispensable, à défaut d'autres méthodes de réduction de ces états rapides, pour l'obtention d'une mobilité effective des électrons du canal importante. Dans une étude prospective non publiée [6], nous avons évalué l'aptitude de $\mathrm{InPO}_{4}$, qui semble être le principal constituant de l'oxyde natif (thermique et plasma) [7], à assurer une bonne stabilité des composants. Les conclusions de cette étude montrent que, compte tenu de ses propriétés électriques et physico-chimiques, cet oxyde a relativement peu de "chances " de réduire les dérives dans des limites compatibles avec un bon fonctionnement des dispositifs.

Dans cet article, nous proposons une nouvelle approche expérimentale de ces phénomènes fondée sur l'étude de la photoémission interne de structures $\mathrm{Al}-\mathrm{Al}_{2} \mathrm{O}_{3}$-InP. Nous verrons que cette technique qui permet, entre autres, la mesure directe de la hauteur de barrière semiconducteur-isolant et l'évaluation de la qualité de l'isolant déposé, constitue un très bon outil pour effectuer le diagnostic de l'origine des phénomènes de dérive.

Le prochain paragraphe est consacré à la description des dispositifs expérimentaux. Les résultats sont présentés et discutés dans le paragraphe 3 ; le paragraphe 4 conclura cet article.

\section{Dispositifs expérimentaux.}

2.1 PréParation DeS ÉChantillons. - Les expériences sont conduites sur des structures MIS/InP dont l'isolant, $\mathrm{Al}_{2} \mathrm{O}_{3}$, est déposé par évaporation au canon à électrons. Avant le dépôt, les substrats d'InP (type $\mathrm{N}, N_{\mathrm{D}} \simeq 1,5 \times 10^{16} \mathrm{at} . / \mathrm{cm}^{-3}$; orientation 100 ) subissent un polissage mécano-chimique $\mathrm{Br}-\mathrm{CH}_{3} \mathrm{OH}$ dont les détails sont décrits dans [5]. Ils sont ensuite soumis à divers traitements chimiques tendant :

- soit à décaper l'oxyde natif en traitant les échantillons dans une solution HF [5] ;

- soit à favoriser la formation d'un oxyde ; dans ce cas, deux démarches ont été suivies :

- oxydation thermique des échantillons (après décapage $\mathrm{HF}$ ) dans $\mathrm{O}_{2}$ à $150^{\circ} \mathrm{C}$ pendant deux heures,

- traitement des échantillons dans une solution $\mathrm{NH}_{4} \mathrm{OH}$ [5], réputée conduire à la constitution d'un oxyde natif stable [8].

Pour comparaison, les mesures de photoémission sont aussi effectuées sur des structures $\mathrm{Al}-\mathrm{Al}_{2} \mathrm{O}_{3}-\mathrm{Si}$ dont l'isolant a été déposé dans les mêmes conditions, après avoir soumis le substrat de silicium à un traitement chimique classique.

2.2 Photó́mission. - Le banc de photoémission constitué d'une lampe xénon (1000 W), d'un monochromateur et d'un système de détection électrométrique solidaire du porte-échantillon (sensibilité $10^{-15} \mathrm{~A}$ ), est piloté par ordinateur et permet le tracé automatique de la réponse spectrale de photoémission interne des porteurs par-dessus les barrières métalisolant (polarisation de grille $V_{\mathrm{G}}<0$ ) et semiconducteur-isolant $\left(V_{G}>0\right)$.

Les hauteurs de barrière sont déterminées par la méthode classique de Fowler $[9,10]$, par extrapolation de la partie linéaire de la caractéristique $Y^{1 / 2}(E)$, où $Y$ est le rendement quantique et $E$ l'énergie des photons incidents [11].

\section{Résultats expérimentaux et discussion.}

3.1 RéPONSES SPECTRAles. - Pour un traitement chimique donné du substrat, on observe une dispersion des courbes de réponse spectrale d'un dépôt à l'autre, essentiellement au voisinage du seuil de photoémission. Ce comportement est illustré figure 1 dans le cas d'une attaque HF du substrat. De plus, les dispersions de la courbe de photoémission du semiconducteur sont parfaitement corrélées à celles de la courbe de photoémission du métal. Par ailleurs, les caractéristiques spectrales obtenues pour un même dépôt sont pratiquement indépendantes du traitement chimique du substrat, ainsi qu'on peut le constater sur la figure 2 où sont tracées les réponses spectrales pour les trois traitements décrits précédemment. La première conclusion que l'on peut déduire de ces observations est que l'allure de la réponse spectrale dépend au premier ordre de l'isolant déposé. De fait, les conditions de fabrication de $\mathrm{Al}_{2} \mathrm{O}_{3}$ ne sont pas strictement reproductibles d'un dépôt à l'autre. En particu- 


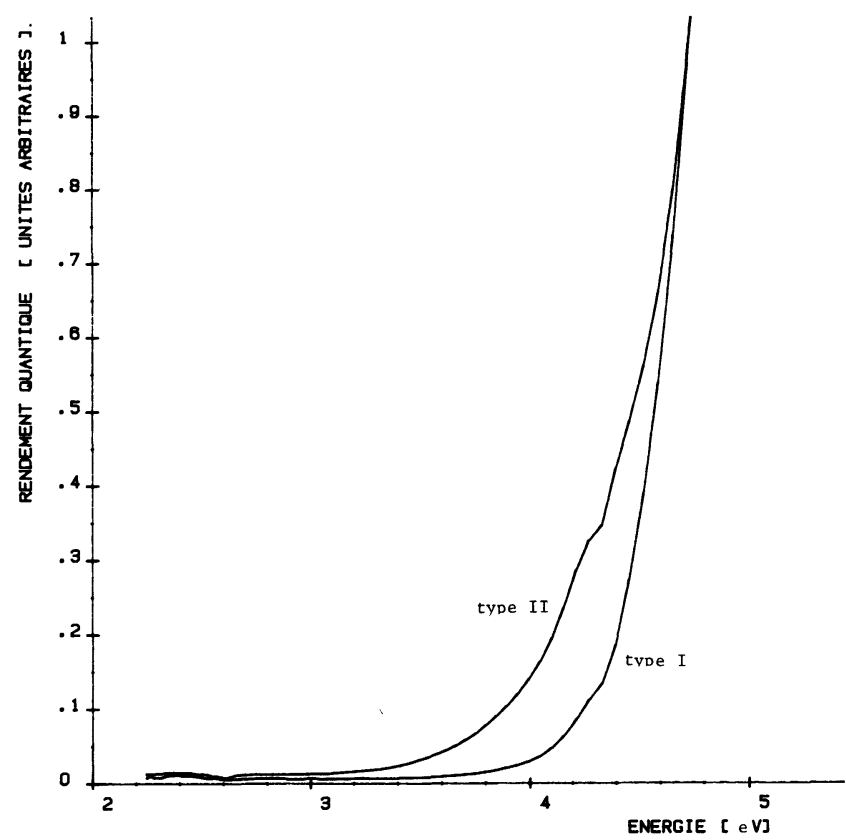

Fig. 1. - Courbes de réponse spectrale de photoémission interne de structures $\mathrm{Al}_{2} \mathrm{O}_{3}-\mathrm{InP}$ dans le cas d'une attaque des substrats d'InP dans une solution à base de HF. On observe une dispersion des caractéristiques d'un dépôt d'isolant à l'autre. Les caractéristiques de type I, qui présentent un seuil bien défini, correspondent à un isolant de "bonne qualité ", contrairement aux caractéristiques de type II.

[Internal photoemission spectral responses of $\mathrm{Al}_{2} \mathrm{O}_{3}$-InP structures for chemically etched InP substrates in $\mathrm{HF}$ solution. A dispersion of the characteristics is observed as a function of the deposition conditions of the insulator. Type I characteristics, with a sharp threshold, are typical of good quality insulators unlike type II characteristics.]

lier, la vitesse de dépôt, dont le contrôle est relativement délicat au canon à électrons, peut fluctuer entre 3 et $10 \AA / \mathrm{s}$. De même, la pression de base peut varier de quelque $10^{-7}$ torr à environ $10^{-6}$ torr.

Ce n'est pas l'objet de ce travail que d'analyser en détail l'influence des conditions de dépôt de $\mathrm{Al}_{2} \mathrm{O}_{3}$ sur la réponse spectrale de photoémission. Nous nous contenterons donc, dans la suite, de distinguer deux catégories de couches d'alumine déposées : la première concerne les isolants présentant un seuil de photoémission bien défini (caractéristiques de type I de la figure 1); la deuxième est relative, au contraire, aux isolants dont le seuil est "mou " et présentant une réponse spectrale décalée vers les énergies plus faibles à faible rendement quantique (caractéristique de type II de la figure 1).

Il existe plusieurs explications possibles de ces différences observées dans les réponses spectrales. Une interprétation vraisemblable consiste à mettre en cause des états électroniques en queue de bande de conduction de $\mathrm{Al}_{2} \mathrm{O}_{3}$. Une forte densité de ces derniers entraînerait un adoucissement de la réponse au voisinage du

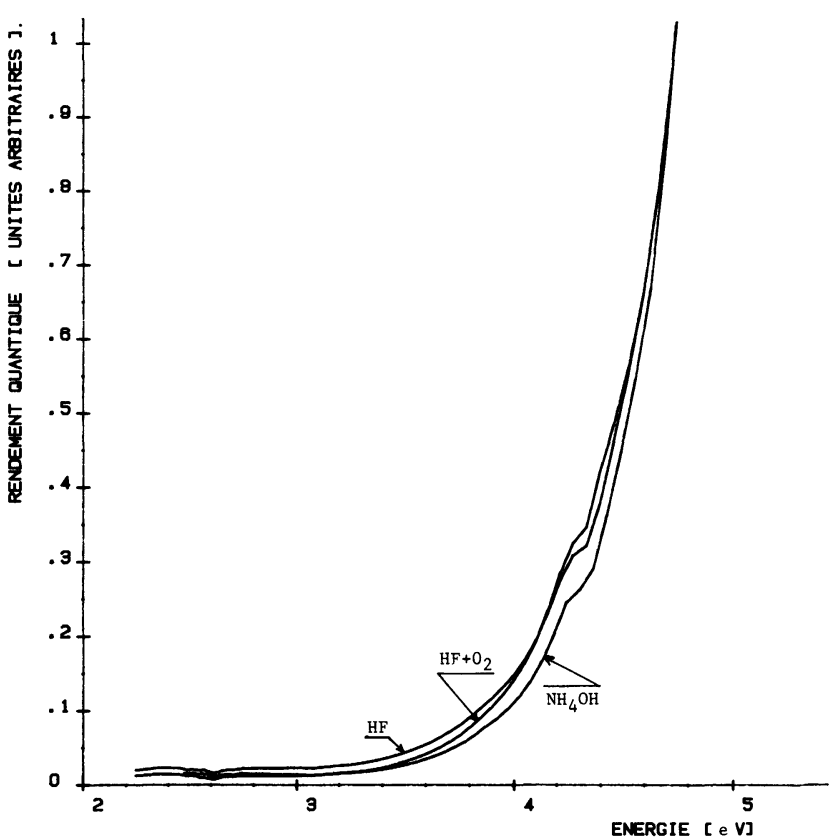

Fig. 2. - Courbes de réponse spectrale de photoémission obtenues pour un même dépôt d'Al $_{2} \mathrm{O}_{3}$ et pour les trois traitements chimiques de substrats décrits dans le texte $\left(\mathrm{NH}_{4} \mathrm{OH}, \mathrm{HF}\right.$ et $\left.\mathrm{HF}+\mathrm{O}_{2}\right)$. On observe peu d'influence du traitement chimique.

[Internal photoemission spectral responses obtained for the same $\mathrm{Al}_{2} \mathrm{O}_{3}$ deposition and for the three chemical treatments described in the text $\left(\mathrm{NH}_{4} \mathrm{OH}, \mathrm{HF}\right.$ and $\mathrm{HF}+\mathrm{O}_{2}$ ). The shape of the curves is merely insensitive to the surface chemical treatment.]

seuil et inversement. Si cette interprétation est correcte, on pourrait en conclure qu'une réponse spectrale raide est la signature d'un isolant de bonne qualité : cette proposition sera renforcée par les résultats expérimentaux présentés dans le paragraphe suivant.

3.2 CORRÉlation ENTRE LES CARACTÉRISTIQUES SPECTRALES ET LA STABILITÉ DES DISPOSITIFS. - La stabilité des dispositifs est évaluée qualitativement sur la base de l'amplitude de l'hystérésis $(\Delta V)$ des caractéristiques capacité-tension $(C(V))$, tracées à la fréquence de $1 \mathrm{MHz}$, le balayage des tensions étant réalisé entre -5 et $+5 \mathrm{~V}$ à la vitesse de $100 \mathrm{mV} / \mathrm{s}$. On observe systématiquement une hystérésis indiquant un piégeage des porteurs à proximité de l'interface. Deux types de comportement sont observés :

- dans le cas où l'isolant déposé possède des caractéristiques de photoémission de type I (Fig. 1), l'amplitude de l'hystérésis $\Delta V$ est pratiquement indépendante du traitement chimique du substrat semiconducteur et se situe dans la gamme $200-500 \mathrm{mV}$,

- dans le cas de caractéristiques spectrales de type II, on observe une différence très marquée entre les échantillons traités dans une solution $\mathrm{NH}_{4} \mathrm{OH}$ pour lesquels $\Delta V$ reste de l'ordre de $200-500 \mathrm{mV}$, et les autres (traitement $\mathrm{HF}$ ou $\mathrm{HF}+$ oxydation) pour lesquels $\Delta V$ s'élève à quelques volts et peut excéder $5 \mathrm{~V}$. 
Les résultats expérimentaux exposés ci-dessus et dans le paragraphe précédent suggèrent les conclusions suivantes :

i) la qualité de l'isolant de grille demeure un facteur essentiel pour la stabilité des composants MIS sur InP dont les phénomènes de dérive ont été jusqu'à présent imputés aux seules propriétés électroniques et physicochimiques de l'interface. Précisons que qualité n'est pas synonyme de forte résistivité. L'alumine du type II, exhibant une caractéristique spectrale "molle" et source d'hystérésis importante, recèle probablement une forte densité de pièges tout en présentant une bonne résistivité $\left(>10^{14} \Omega . \mathrm{cm}\right)$;

ii) le rôle favorable d'un oxyde natif stable (traitement $\mathrm{NH}_{4} \mathrm{OH}$ ) pour la réduction des dérives est mis en lumière en ce sens qu'il est capable d'assurer une protection efficace non pas contre des phénomènes de dérive intrinsèques issus de l'interface, mais contre ceux, extrinsèques, induits par un isolant de mauvaise qualité. Cette protection est à mettre au crédit de la barrière tunnel supplémentaire qu'oppose l'oxyde de surface à la communication des porteurs du semiconducteur et des pièges de l'isolant déposé. Bien que, comme nous l'avons signalé plus haut, les réponses spectrales de photoémission dépendent peu du traitement chimique du substrat, on remarque cependant que l'échantillon traité $\mathrm{NH}_{4} \mathrm{OH}$ (Fig. 2) présente une caractéristique légèrement décalée vers les énergies plus élevées, ce qui indique une barrière effective légèrement supérieure dans ce cas. Cette différence reste très faible dans la mesure où, dans l'expérience de photoémission, les électrons sont émis par-dessus la barrière $\mathrm{Al}_{2} \mathrm{O}_{3} / \mathrm{InP}$, et donc vraisemblablement aussi par-dessus la barrière tunnel de l'oxyde.

3. 3 HAUTEURS DE BARRIÈRE. - La hauteur de barrière $\chi_{n}$, présentée aux électrons de conduction de l'InP par l'alumine évaporée au canon à électrons est égale à $2,8 \pm 0,1 \mathrm{eV} \cdot \chi_{\mathrm{n}}$ est pratiquement insensible au traitement chimique des substrats InP. Des mesures similaires effectuées sur des structures métal- $\mathrm{Al}_{2} \mathrm{O}_{3}-\mathrm{Si}$, fabriquées simultanément, on déduit une valeur de $\chi_{\mathrm{n}}$ égale à $3,1 \pm 0,1 \mathrm{eV}$ dans le cas du silicium. La comparaison de ces résultats avec les valeurs rapportées dans la littérature de l'affinité électronique de l'InP et $\mathrm{du} \mathrm{Si}$, indique une contribution dipolaire relative de $0,65 \pm 0,1 \mathrm{eV}$ à l'interface $\mathrm{Al}_{2} \mathrm{O}_{3}$-InP par rapport à l'interface $\mathrm{Al}_{2} \mathrm{O}_{3}-\mathrm{Si}$. Ces résultats sont illustrés figure 3 où sont représentés les diagrammes de bande des deux systèmes isolant-semiconducteur. La différence observée $(0,3 \pm 0,1 \mathrm{eV})$ entre les valeurs de $\chi_{\mathrm{n}}$ pour l'InP et le Si n'est pas suffisante pour expliquer les phénomènes de dérive observés dans les structures MIS-InP durant leur fonctionnement normal, sur la base de processus d'injection de porteurs chauds par-dessus la barrière $\mathrm{Al}_{2} \mathrm{O}_{3}$-InP, suivis de leur piégeage à l'intérieur de l'alumine.

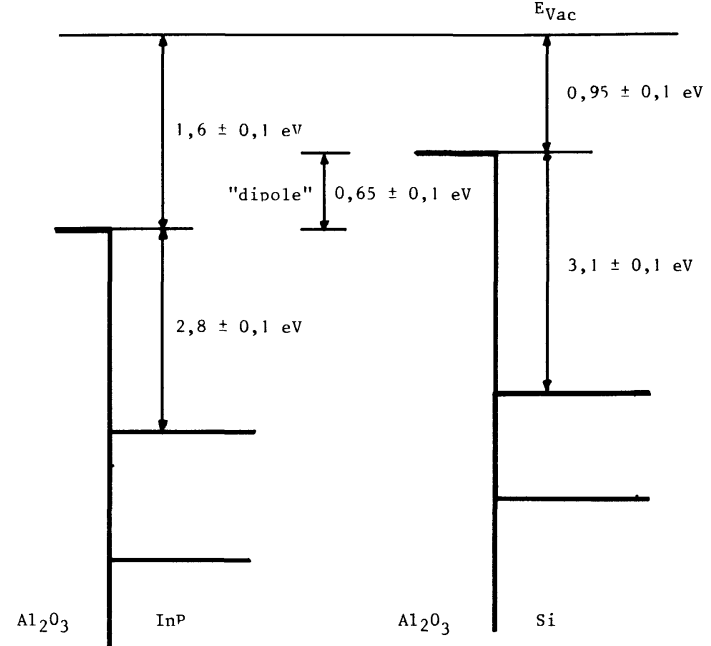

Fig. 3. - Diagrammes des bandes d'énergie des systèmes $\mathrm{Al}_{2} \mathrm{O}_{3}$-InP et $\mathrm{Al}_{2} \mathrm{O}_{3}$-Si.

[Energy band diagrams of $\mathrm{Al}_{2} \mathrm{O}_{3}-\mathrm{InP}$ and $\mathrm{Al}_{2} \mathrm{O}_{3}$-Si systems.]

Signalons enfin que les mesures de photoémission effectuées sur des structures soumises à une illumination conduisent à la même valeur de $\chi_{\mathrm{n}}$ qui est relativement insensible aux contraintes électriques subies par le dispositif (par exemple photo-injection de porteurs sous polarisation). Cela signifie que l'interface ou sa proximité immédiate ne saurait être le siège d'un piégeage intense de porteurs.

\section{Conclusions.}

L'origine des phénomènes d'instabilité observés dans les structures MIS-InP est incertaine et leur étude sur la seule base de l'analyse des caractéristiques électriques de ces dispositifs ne permet pas de lever les ambiguiités qui subsistent. Nous avons montré dans cet article que la technique de photoémission interne permet d'obtenir des compléments d'information autorisant un diagnostic beaucoup plus sûr de l'origine des dérives. C'est ainsi que la différence observée $(0,3 \pm 0,1 \mathrm{eV})$ entre les hauteurs de barrière $\chi_{n}$ présentées par $\mathrm{Al}_{2} \mathrm{O}_{3}$ aux électrons de conduction de l'InP $(2,8 \pm 0,1 \mathrm{eV})$ et du $\mathrm{Si}(3,1 \pm 0,1 \mathrm{eV})$ n'est pas suffisante pour expliquer les phénomènes de dérive présents dans les structures MIS-InP, sur la base de processus d'injection de porteurs chauds suivis de leur piégeage dans l'alumine. Nous avons en outre établi que les dérives des composants peuvent être fortement influencées par les propriétés de l'isolant déposé. A cet égard, la constitution d'un oxyde natif stable (attaque chimique du substrat dans une solution à base de $\mathrm{NH}_{4} \mathrm{OH}$ ) protège la surface du semiconducteur contre les dérives que pourraient induire, en son absence, un isolant déposé de mauvaise qualité, en 
limitant la communication entre les pièges de ce dernier et les porteurs du semiconducteur. En d'autres termes, la présence d'un oxyde natif stable permet d'éviter la dispersion des caractéristiques de dérive pouvant résulter d'une mauvaise reproductibilité des conditions de fabrication de l'isolant déposé. Bien que la constitution d'un oxyde natif stable ne soit probablement pas susceptible d'assurer une stabilité suffisante de la structure MIS [6], il semble qu'en l'état de l'art actuel, ce soit la seule solution applicable, car elle permet à la fois de neutraliser les états rapides de surface [5] et de limiter les instabilités, auxquelles elle assure un caractère... reproductible.

\section{Remerciements.}

Les auteurs remercient vivement Mrs J. L. Perrossier et C. Santinelli pour leur participation à la fabrication des échantillons, de même que Mr Garrigues pour sa lecture critique du manuscrit. Le Centre National d'Etude des Télécommunications de Lannion est également remercié pour la fourniture des substrats d'InP utilisés dans cette étude. Cette recherche était conduite dans le cadre du GRECO «Physique et Technologie des Dispositifs à Semiconducteurs Composés III-V » et d'un contrat DAII no 82.35.178.00.790. 75.00.19.

\section{Bibliographie}

[1] Lile, D. L. et Collins, D. A., Appl. Phys. Lett. 28 (1976) 554.

[2] OKamura, M. et Kobayashi, T., Japan. J. Appl. Phys. 19 (1980) 2151.

[3] Okamura, M. et Kobayashi, T., Electron. Lett. 17 (1981) 941.

[4] Lile, D. L. et Taylor, M. J., J. Appl. Phys. 54 (1983) 260.

[5] Sautreuil, B., Bailly, B., Blanchet, R., Garrigues, $M$. et VikTorovitch, $P$., ce même numéro.

[6] Viktorovitch, P., Note interne. Laboratoire d'Elec- tronique de l'Ecole Centrale de Lyon, non publiée (1983).

[7] Wager, J. F., Makky, W. H., Wilmsen, C. W. et Meiners, L. G., Thin Solid Films 95 (1982) 343.

[8] Guivarc'h, A., L'Haridon, H., Pelous, G., HolLINGer, G. et Pertosa, P., à paraître dans J. Appl. Phys.

[9] Fowler, R. H., Phys. Rev. 38 (1931) 45.

[10] Deal, B. E., Snow, E. H. et Mead, C. A., J. Phys. Chem. Solids 27 (1966) 1873.

[11] Williams, R., Phys. Rev. A 140 (1965) 569. 